\title{
Horrorszenario
}

\section{Herztransplantation nach nicht indizierter Angiografie}

\author{
Eine gleichermaßen ungewöhnliche \\ wie bewegende Kasuistik lehrt einmal \\ mehr, dass der Nutzen ärztlicher Maß- \\ nahmen und deren potenzielle Folgen \\ bei jedem Patienten sorgfältig bedacht \\ werden müssen.
}

Eine 52-jährige Krankenschwester berichtete über seit zwei Tagen anhaltende, stechende, belastungsunabhängige Brustschmerzen, die sich durch Heben des rechten Arms und tiefe Inspiration verstärkten. Sie hatte einige Wochen vorher wegen milder Hypertonie und leichtem Übergewicht mit einer Diät und einem Fitnessprogramm begonnen.

Die Untersuchung war unauffällig. Die bekannten Schmerzen konnten durch Anheben des rechten Arms und Palpation der rechten Thoraxseite ausgelöst werden. Die Lipid- und CRP-Spiegel waren normal. Die Verdachtsdiagnose lautete: atypische Brustschmerzen, am ehesten muskuloskeletaler Ursache.

Trotzdem ordnete der Hausarzt eine kardiale CT-Angiografie (CCTA) an. Dabei wurden in der A. circumflexa und der dominanten rechten Koronararterie leichte, nicht einengende, nicht kalzifizierte Pla- ques gesehen. Im proximalen Ramus interventricularis anterior erschwerten Verkalkungen eine zuverlässige Quantifizierung der Lumeneinengung. Die folgende Koronarangiografie zeigte aber nur milde luminale Irregularitäten im Ramus interventricularis anterior.

Kurz nach der zweiten Kontrastmittelinjektion traten intensive Herzschmerzen zusammen mit Hypotonie und Tachykardie auf. Ein Aortogramm zeigte eine Dissektion der linken Hauptstammarterie, die eine aortale Ballonpumpe und einen Bypass erforderten.

Sechs Wochen nach der Entlassung traten schwere Brustschmerzen auf. Wegen eines Bypassverschlusses erfolgten Dilatation und Stenteinlagen. Trotz einer adäquaten antithrombotischen Therapie trat acht Wochen später ein Herzinfarkt mit einem therapierefraktären kardiogenen Schock auf, der notfallmäßig eine Herztransplantation erzwang.

II Kommentar: Der Hausarzt entschied sich für die CCTA, um „eine Koronarstenose sicher auszuschließen und die Patientin beruhigen zu können “. Nach den Leitlinien der Fachgesellschaften war die CCTA bei dieser Patientin, die ein niedriges kardiovaskuläres Risiko und untypische Brustschmerzen hatte, nicht indiziert. Denn die Vortestwahrscheinlichkeit einer nennenswerten koronaren Herzerkrankung, d.h. des Risikos für eine tödliche oder nicht tödliche koronare Komplikation innerhalb der folgenden zehn Jahre lag unter $10 \%$.

Der falsch positive bzw. unklare CCTABefund am Ramus interventricularis anterior führte zur ebenfalls unnötigen Folgeuntersuchung, der Koronarangiografie mit der Serie verheerender Komplikationen. Die Komplikationsrate der Koronarangiografie ist zwar gering, aber katastrophale Folgen sind bekannt. Für die Beruhigung und Versicherung ängstlicher Patienten mit geringer Krankheitswahrscheinlichkeit ist nicht eine komplikationsträchtige und kostspielige Diagnostik geeignet, sondern das ärztliche Gespräch.

Prof. Dr. med. Heinrich Holzgreve, München I"

II Becker MC et al. Left main trunk coronary artery dissection as a consequence of inaccurate coronary computed tomographic angiography. Arch Intern Med doi:10.1001/ archinternmed.2010.464

\section{Hämorrhagischer Schlaganfall}

\section{Mögliche Kontraindikation für Statintherapie?}

Bei Patienten, die einen hämorrhagischen Schlaganfall hatten, schadet eine Statintherapie wahrscheinlich mehr als sie nutzt.

Um den Nutzen und die Risiken einer Statintherapie nach einer Hirnblutung gegeneinander abzuwägen, haben die Autoren die Ergebnisse publizierter Studien zur Statintherapie und zum Rezidivrisiko nach Hirnblutungen in ein Markov-Entscheidungsmodell eingegeben. Für verschiedene klinische Szenarien wurde statistisch mo- delliert, wie sich eine Statintherapie auf die Lebenserwartung in qualitätsadjustierten Lebensjahren (QALY) auswirkt. Dabei wurde der Einfluss von Veränderungen der Blutungslokalisation, des kardiovaskulären Risikos und des mit der Statintherapie assoziierten Blutungsrisiko simuliert.

Zunächst wurde der theoretische Fall eines 65-jährigen Mannes mit einer lobären, in einem der Hirnlappen gelegenen intrazerebralen Blutung und ohne vorherige ischämische kardiovaskuläre Ereignisse zugrunde gelegt. In dieser Konstella- tion führte der Verzicht auf eine Statintherapie zu einer Lebensverlängerung um 2,2 OALY.

Patienten mit einer tiefer gelegenen Hirnblutung, z.B. einer hypertensiven Stammganglienblutung, profitierten ebenfalls nicht von einer Statintherapie, sie hatten jedoch einen niedrigeren Nettonutzen (o,8 OALY) durch den Therapieverzicht.

In Sensitivitätsanalysen blieb der Nettonutzen durch einen Therapieverzicht erhalten, auch wenn das mit der Therapie assoziierte Blutungsrisiko niedriger oder 